\title{
EDITORIAL
}

\section{It is time for patients to undergo bronchoscopy without discomfort}

\author{
S. Gasparini
}

$\mathbf{T}$ hirty years ago, when I started performing bronchoscopy, sedation for flexible bronchoscopy was not a very common practice. At that time, some authors suggested that routine sedation could do little for patients undergoing the procedure and that bronchoscopy could be carried out without pre-medication [1-3]. However, over the last few decades, several things have changed. Patients' need for comfort, and the desire to avoid anxiety and distress in patients undergoing medical procedures has increased generally. Furthermore, the advent and routine use of new bronchoscopic techniques and technologies have made diagnostic bronchoscopy a more complex procedure that requires prolonged time, the absence of cough, and patient cooperation and comfort. In this new scenario, the problem of sedation during bronchoscopy requires careful re-evaluation in order to definitively assess its indication and also to define the best sedative drugs to be used.

The British Thoracic Society guidelines on diagnostic flexible bronchoscopy recommend that sedation should be offered to patients when there is no contraindication [4], and in surveys conducted in the USA and in the UK in 1999 and in 2002, respectively, only 4 and $10 \%$ of physicians performed bronchoscopy without sedation $[5,6]$. The old argument that up to half of the major complications of bronchoscopy are sedation related [7] is not confirmed by several more recent studies. In fact, even after comparing different sedative drugs versus placebo, no difference in the complication rate between sedated and nonsedated patients has been found [8-14], except for a slight but manageable increase in hypoxaemia risk with the use of midazolam and alfentanil [14] and a prolonged hospital stay after the procedure [10]. In fact, the use of sedation, when compared with placebo, resulted in improved overall tolerance and patient perception of bronchoscopy [9-14], reduced cough $[8,12,14]$, and better predisposition of patients to repeating the procedure [10, 12-14]. Furthermore, sedation has been associated with fewer difficulties for the bronchoscopist and with shortening of the duration of the procedure [13]. Nowadays, on the basis of this evidence, bronchoscopy without sedation could possibly be considered unethical, especially in cases where complex and time-consuming procedures are planned.

The drug or combination of drugs to be used for sedation in flexible bronchoscopy should be able to provide a condition of moderate sedation/analgesia (conscious sedation) that allays anxiety and reduces stress, improves patient comfort, reduces

CORRESPONDENCE: S. Gasparini, SOD Pneumologia, Azienda Ospedaliero-Universitaria "Ospedali Riuniti", Via Conca, 71, 60020 Ancona, Italy. E-mail: s.gasparini@fastnet.it pain and cough, provides amnesia without any risks to ventilatory function or airway patency. The state of conscious sedation is defined by the presence of a purposeful response to verbal or tactile stimulation and adequately preserved spontaneous ventilation without any need for intervention to maintain a patent airway. Deep sedation/analgesia, which should be avoided during flexible bronchoscopy, is characterised by a purposeful response after repeated or painful stimulation, spontaneous ventilation that may be inadequate and a possible need for intervention to maintain a patent airway [15].

Over the years, bronchoscopists have used several drugs, alone or in combination, for sedation, but the majority of studies have evaluated benzodiazepines (mainly diazepam and midazolam) and narcotics (mainly opioids, such as fentanyl and alfentanil), or combinations of these [16-20]. The combination of the sedative and amnesic effects of benzodiazepines with the analgesic and the antitussive actions of opioids is able to provide effective conscious sedation [15], and this is probably the most commonly used regimen in bronchoscopy [21], even if there are not many studies that compared the use of a single drug with a combination (and those that did gave controversial results). In a study on 103 patients, randomised into three arms receiving midazolam alone, alfentanil alone or a combination of the two drugs, GreIG et al. [16] were unable to find any significant difference in the assessment of discomfort between groups, while alfentanil showed a better antitussive efficacy than midazolam. The combination of alfentanil and midazolam did not provide any better antitussive effect, while it caused more desaturations in comparison with alfentanil alone. In another study on 120 patients randomised to receive midazolam and $5 \mathrm{mg}$ intravenous hydrocodone, or midazolam and placebo, perception of cough by both bronchoscopist and nurse was significantly lower in the hydrocodone group. Patient tolerance was also significantly better with hydrocodone than with the placebo. The association did not cause a greater fall in oxygen saturation that midazolam alone [18].

The status of conscious sedation can also be obtained by using propofol, a sedative-hypnotic agent that has a rapid onset and short duration of action. It produces a dose-dependent level of sedation, varying from conscious sedation to general anaesthesia. There is growing evidence in the literature on the advantages of propofol in comparison with benzodiazepines and opioids. Propofol seems to be able to provide a faster onset of action and a reduction in recovery time than midazolam [22-24]. In another study, comparing propofol with diazepam plus fentanyl, the authors were unable to find significant differences in recovery 
between groups, but the patients in the propofol group were more sedated and respiratory frequency decreased only in the diazepam group [25]. In a study on 84 patients randomised to receive midazolam or propofol, patient tolerance was significantly better in the propofol group, whereas the operator's assessment was comparable in both groups. Recovery time was shorter with propofol and no differences in oxygen desaturation were observed between the groups [26].

In the current issue of the European Respiratory Journal (ERJ), SCHLATTER et al. [27] report the results of a prospective, randomised, placebo-controlled study performed on 300 consecutive patients to evaluate whether the combination of propofol and hydrocodone is better than propofol alone at suppressing cough during diagnostic flexible bronchoscopy. The patients were randomised to receive either $4 \mathrm{mg}$ i.v. hydrocodone or placebo. Propofol was administered to all the patients using an intermittent bolus technique, starting with an initial 10-20 mg dose followed by additional boluses of 10$20 \mathrm{mg}$, depending on the clinical effect, in order to maintain the required level of conscious sedation. The combination of propofol and hydrocodone resulted in a significantly lower cough score as evaluated by the physician, nursing staff and patient. Patients' discomfort scores $2 \mathrm{~h}$ after the procedure were also lower in the hydrocodone group. Furthermore, the group receiving the opiate required significantly less propofol for sedation. No differences in complication rate, particularly oxygen desaturation and haemodynamic changes, were observed in the two groups. It is interesting to observe that the advantages of the combination of propofol plus hydrocodone were more evident for all the evaluated parameters in the patients undergoing complex procedures, while no significant differences were obtained for patients who underwent only simple procedures (inspection or bronchial washing). The authors conclude that the combination of propofol and hydrocodone is safe, and that it could be adopted as a standard sedation regimen for flexible bronchoscopy, especially in patients undergoing complex procedures.

The choice of hydrocodone over other opiates is justified by the authors because of its low cost. Unfortunately, i.v. hydrocodone is not commercially available in all countries, and this is the reason why fentanyl or alfentanil are more frequently employed in bronchoscopic practice.

The study by SCHLATTER et al. [27] provides further evidence that propofol is effective for conscious sedation and that it can be used safely even when administered by nonanaesthetists. However, propofol is more expensive than midazolam and its use by nonanaesthetist physicians is not allowed in several countries. These facts may limit its widespread diffusion. Furthermore, since propofol produces a dose-dependent level of sedation, varying from moderate sedation to general anaesthesia, and there is a wide variability in the response of the patients, physicians administering this drug should be qualified to rescue patients from any level of sedation, including general anaesthesia [15]. The practice guidelines for sedation and analgesia by nonanaesthetists, published by the American Society of Anesthesiologists, recommend that an individual other than the person performing the procedure should be present in the room in order to continuously monitor the patient's condition, whatever drug is used for sedation.
This individual should understand the pharmacology of the agents that are administered, be trained to recognise complications associated with sedation and have knowledge of advanced life support [15]. It is curious to see that even if pulmonologists are advantaged by their expertise in the management of the airways, hypoxaemia and ventilation, gastroenterologists are more familiar with the use of propofol for sedation during gastrointestinal endoscopy. The worldwide safety experience of endoscopist-administered propofol exceeds 460,000 patients [28] and gastroenterological scientific associations have even published an official position statement to provide an evidence-based assessment of propofol-mediated sedation by properly trained gastroenterologists [29].

We hope that a document on sedation for bronchoscopy will be published by respiratory scientific societies. Furthermore, thanks to the study by SCHLATTER et al. [27] published in this issue of the $E R J$, there is now adequate evidence in this field to allow an official statement and recommendations that would be a great help to the daily activity of all interventional pulmonologists.

A long time has passed since 1915, when one of the fathers of bronchoscopy, Chevalier Jackson, wrote: "The operator who can keep his patient free from apprehension and who can keep his patient's mind fixed on the task of breathing slowly, deeply, and regularly will get along without any anaesthetic and do better work than another operator under profound general anaesthesia" [30]. Even if the ideal sedation regimen has not been identified yet, today we have different and alternative drugs that can be used effectively and safely. The time has come for our patients to undergo bronchoscopy without any discomfort.

\section{STATEMENT OF INTEREST}

None declared.

\section{REFERENCES}

1 Banerjee A, Banerjee SN, Nachiappan M. Premedication for fibreoptic bronchoscopy (is sedation a must?). Indian J Chest Dis Allied Sci 1986; 28: 76-80.

2 Colt HG, Morris JF. Fiberoptic bronchoscopy without premedication: a retrospective study. Chest 1990; 98: 1327-1330.

3 Hatton MQF, Allen MB, Vathenen AS, et al. Does sedation help in fibreoptic bronchoscopy? BMJ 1994; 309: 1206.

4 British Thoracic Society Bronchoscopy Guidelines Committee, a SubComitte of the Standards of Care Committee at the British Thoracic Society. British Thoracic Society guidelines on diagnostic flexible bronchoscopy. Thorax 2001; 56: Suppl. 1, i1-i21.

5 Colt HG, Prakash UBS, Offord KP. Bronchoscopy in North America: survey by the American Association for Bronchology, 1999. J Bronchol 2000; 7: 8-25.

6 Smyth CM, Stead RJ. Survey of flexible fibreoptic bronchoscopy in the United Kindom. Eur Respir J 2002; 19: 458-463.

7 Credle WF, Smiddy JF, Elliot RC. Complications of fibreoptic bronchoscopy. Am Rev Respir Dis 1976; 109: 67-72.

8 Rees PJ, Webb JR. Premedication for fibreoptic bronchoscopy. Thorax 1983; 38: 624-627.

9 Maltais F, Laberge F, Laviolette M. A randomized, double-blind, placebo controlled study of lorazepam as premedication for bronchoscopy. Chest 1996; 109: 1195-1198.

10 Maguire G, Rubinfeld AR. Patients prefer sedation for fibreoptic bronchoscopy. Respirology 1998; 3: 81-85. 
11 Putinati S, Ballerin L, Corbetta L, et al. Patient satisfaction with conscious sedation for bronchoscopy. Chest 1999; 115: 1437-1440.

12 Gonzalez R. Should patients undergoing a bronchoscopy be sedated? Acta Anaesthesiol Scand 2003; 47: 411-415.

13 Cases Viedma E. A randomized study of midazolam for sedation in flexible bronchoscopy. Arch Bronchopneumol 2010; 46: 302-309.

$14 \mathrm{Ni}$ YL. Conscious sedation reduces patient discomfort and improves satisfaction in flexible bronchoscopy. Chang Gung Med J 2010; 33: 443-452.

15 American Society of Anesthesiologists. Practice guidelines for sedation and analgesia by non-anesthesiologists. Anesthesiology 2002; 96: 1004-1017.

16 Greig JH, Cooper SM, Kasimbazi HJN, et al. Sedation for fibreoptic bronchoscopy. Respir Med 1995; 89: 53-56.

17 Williams TJ. Midazolam sedation to produce complete amnesia for bronchoscopy: 2 years' experience at district general hospital. Respir Med 1999; 93: 361-365.

18 Stolz D, Chhajed PN, Leuppi JD, et al. Cough suppression during flexible bronchoscopy using combined sedation with midazolam and hydrocodone: a randomized, double blind, placebo controlled trial. Thorax 2004; 59: 773-776.

19 Chhajed PN, Wallner J, Stolz D, et al. Sedative drug requirements during flexible bronchoscopy. Respiration 2005; 72: 617-621.

20 Dreher M. Sedation during flexible bronchoscopy in patients with pre-existing respiratory failure: midazolam versus midazolam plus alfentanil. Respiration 2010; 79: 307-314.

21 Jantz MA. The old and the new of sedation for bronchoscopy. Chest 2009; 135: 4-6.
22 Crawford M, Pollock J, Anderson K, et al. Comparison of midazolam with propofol for sedation in outpatient bronchoscopy. Br J Anaesth 1992; 70: 419-422.

23 Clarkson $\mathrm{K}$, Power $\mathrm{CK}, \mathrm{O}^{\prime}$ Connell F, et al. Comparative evaluation of propofol and midazolam as sedative agents in fiberoptic bronchoscopy. Chest 1993; 104: 1029-1031.

24 Stolz D, Kurer G, Meyer A, et al. Propofol versus combined sedation in flexible bronchoscopy: a randomised non-inferiority trial. Eur Respir J 2009; 34: 1024-1030.

25 Randell T. Sedation for bronchofiberoscopy: comparison between propofol infusion and intravenous boluses of fentanyl and diazepam. Acta Anaesthesiol Scand 1992; 36: 221-225.

26 Clark G, Licker M, Younossian AB, et al. Titrated sedation with propofol or midazolam for flexible bronchoscopy: a randomised trial. Eur Respir J 2009; 34: 1277-1283.

27 Schlatter L, Pflimlin E, Fehrke B, et al. Propofol versus propofol plus hydrocodone for flexible bronchoscopy: a randomised study. Eur Respir J 2011; 38: 529-537.

28 Deenadayalu VP, Eid EF, Goff JS, et al. Non-anesthesiologist administered propofol sedation for endoscopic procedure: a worldwide safety review. Gastrointest Endosc 2008; 67: AB107.

29 Vargo JJ, Cohen LB, Rex DK, et al. Position statement: nonanesthesiologist administration of propofol for GI endoscopy. Gastrointest Endosc 2009; 70: 1053-1059.

30 Jackson C. Anaesthesia for peroral endoscopy. In: Jackson C, ed. Peroral Endoscopy and Laryngeal Surgery. St Louis, The Laryngoscope Company Publishers, 1915; pp. 54-72. 